\title{
A High Sensitive Sensor Using Mim Coupled With a Rectangular Cavity Based on Fano Resonance
}

hocine bahri ( $\square$ hocine.microelec@gmail.com )

Université Larbi Tébessi - Tébessa Faculté des Sciences et de la Technologie: Universite Larbi Tebessi -

Tebessa Faculte des Sciences et de la Technologie https://orcid.org/0000-0003-0932-5533

\section{Souheil Mouetsi}

University of Larbi Tebessi - Tebessa Faculty of Science and Technology: Universite Larbi Tebessi -

Tebessa Faculte des Sciences et de la Technologie

Abdesselam Hocini

University of M'sila: Universite de M'sila

hocine Ben Salah

University of M'sila: Universite de M'sila

\section{Research Article}

Keywords: Surface Plasmon Polaritons (SPP), Metal-Insulator-metal (MIM) waveguide, Fano resonance, refractive index, sensor

Posted Date: March 17th, 2021

DOI: https://doi.org/10.21203/rs.3.rs-292641/v1

License: (c) (1) This work is licensed under a Creative Commons Attribution 4.0 International License.

Read Full License 


\title{
A high sensitive sensor using MIM coupled with a rectangular cavity based on Fano resonance
}

\author{
Hocine Bahri' ${ }^{\text {(D) }}$. Souheil Mouetsi ${ }^{1}$ • Abdesselam \\ Hocini' $^{(1)}$ - Hocine Ben salah²
}

Received: date / Accepted: date

\begin{abstract}
In this paper, a design with high sensitivity of a plasmonic biosensor by waveguide system is proposed, based on Metal-Insulator-Metal (MIM) coupled with unique rectangular cavities, this structure numerically simulated using the Finite-Difference Time-Domain method (FDTD) in two Dimensions (2D), and analyzed for the optimal sensor performance, by detecting the resonance wavelength and varying the refractive index (RI). The results show two sharp transmission peaks with high transmittance and asymmetrical line-shaped Fano resonances achieved with high value of sensitivity is $3010 \mathrm{~nm} / \mathrm{RIU}$, by taking the wavelength resolution reach as high as $3.84 \times 10-6$ RIU. Considering the standards of Chip-scale integrated planar photonic sensing, the newly designed of the proposed structure with such high sensitivity provides remarkable properties suitable for biosensors, filter, and provide a new possibility for designing compact and high-performance plasmonic biosensors devices.
\end{abstract}

Keywords Surface Plasmon Polaritons (SPP) - Metal-Insulator-metal (MIM) waveguide $\cdot$ Fano resonance $\cdot$ refractive index $\cdot$ sensor

\author{
Hocine Bahri \\ bahri.hocine@univ-oeb.dz \\ Souheil Mouetsi \\ souhei125m@gmail.com \\ Abdesselam Hocini \\ abdesselam.hocini@univ- \\ msila.dz \\ Hocine Ben salah \\ hocine.bensalah@univ- \\ msila.dz \\ 1 Department of electrical engineering, faculty of sciences and applied sciences, \\ University Larbi Ben M’ hidi,Oum El Bouaghi, Algeria. \\ 2 Laboratoire d' Analyse des Signaux et Syst'emes, Department of \\ Electronics, Univer- sity of M’ Sila, BP.166, Route Ichebilia, M’ sila, \\ 28000 Algeria \\ 3 University yahia fares médéa, Algeria
}




\section{Introduction}

Electromagnetic waves coupled to collective oscillations of free electrons in a metal, known as surface plasmon polaritons (SPPs), its regarded as the most promising way to realize highly integrated optical circuits and has attracted great attention in recent years(Dionne et al. 2006). Many Metal-Insulator-Metal (MIM) plasmonic devices based on SPPs have been recently introduced, some of them include plasmonic filters(Hocini et al. 2020), sensors(Ben salah et al. 2019), splitters(Xiang, D. Li 2014), switches(Zhao, W. Lu 2011), etc...

Although MIM has more transmission loss, it can be neglected in nanoscale devices(Han, Z. Liu, L. Forsberg 2006). As a fundamental resonant effect, the Fano resonance, which arises from the interference between a localized state and a continuum band(Fano 1961), has been widely known in numerous physic systems, such as metamaterials(Huang et al. 2012), a metallic nanodisk(Ren et al. 2013). Different from the Lorentzian resonance, the Fano resonance exhibits a typically sharp and asymmetric line profile(Miroshnichenko et al. 2010), which has great important applications in demultiplexing(Ben salah et al. 2019), plasmonic switch(Reza and Mansouribirjandi 2018), and so on. Fano resonance profile promises a new application in sensors. Thus, a new possibility of optical components using ultra-compact plasmonic structure realizing Fano resonance in highly integrated optics(Yi et al. 2018).

In this paper, two sharp Fano resonances are achieved by employing rectangular cavities, we placed a perpendicular cavity between the MIM waveguides, while another horizontal one is integrated to the bottom of the first one. Due to the interactions, the Fano resonances dual with asymmetrical line shapes; wherefore, double sharp peaks are obtained with high sensitivity. Besides, to enhance the sensitivity an additional cavity is added to the middle of the perpendicular cavity. By using the finite-difference time-domain (FDTD) method the performances of the structure are investigated, and it is believed that the proposed structure can pave applications in the optical biosensor area, furthermore due to their simplicity and small dimension we can realizing nanoscale for the optical device.

\section{Analysis and design}

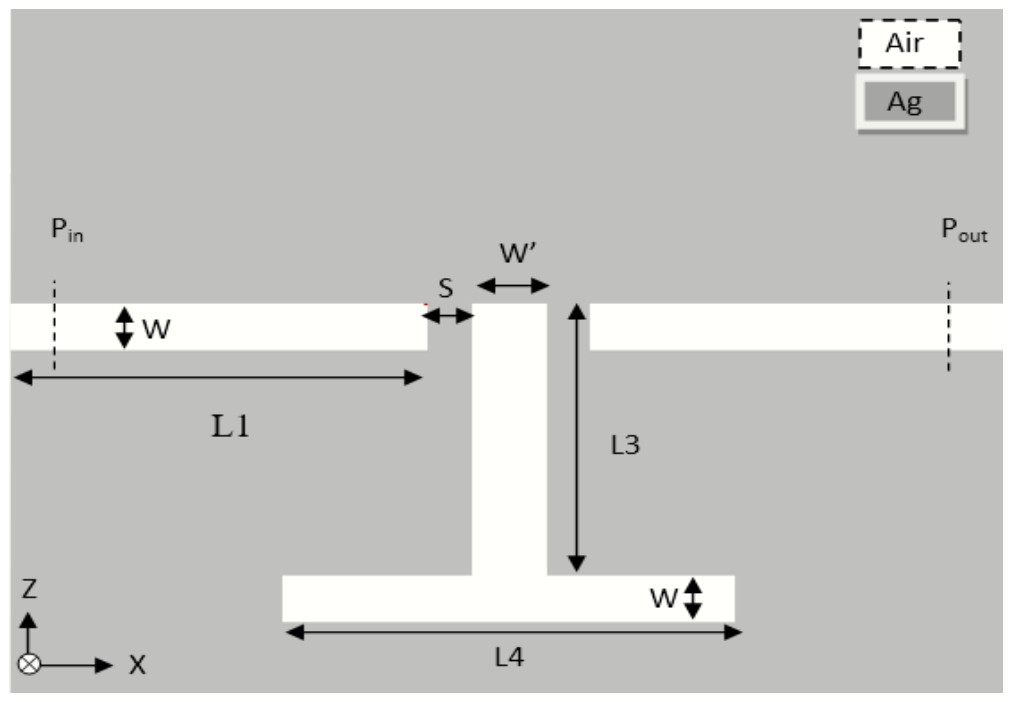

Fig. $12 \mathrm{D}$ schematic diagram of the proposed sensor structure.

The schematic views of the proposed sensor structure with discrete localized and continuum band of state, this asymmetric Fano and symmetric Lorentzian shapes occurs the Fano resonance, and it described by the famous Fano formula(U. Fano 1961):

$$
\sigma(\mathrm{E})=\mathrm{D}^{2} \frac{(q+\Omega)^{2}}{1+\Omega^{2}}
$$

where $\mathrm{E}$ is the energy, $\mathrm{q}=\cot \delta$ is Fano parameter, the phase shift $\delta$ of the continuum states, $\Omega=2\left(\mathrm{E}^{-} \mathrm{E} 0\right) / \Gamma$, where $\Gamma$ and $\mathrm{E} 0$ are the resonance width and energy, respectively, and $\mathrm{D}^{2}=4 \sin ^{2} \delta$ 
Equation (1) is generally applicable for absorption and different optical spectra in a diversity of systems. Recently a lot of interest in the Fano in photonics. Actually, we can treat almost any resonant as quasi-discrete with a complex frequency that can be described in terms of Fano resonance.

Fig. 1 shows the schematic diagram structure with tow rectangular cavity A and B (both named as T cavity), the vertical slot cavity named as cavity A with a length of L3 placed between two MIM waveguides with a length of L2 symmetrically with coupling distance S. B integrated on the bottom of the cavity A with a length of L4. the gray and white area represents Silver is considered as a noble metal due to its low absorption property(Reza and Mansouri-birjandi 2018), and dielectric (Air), respectively. Besides, The relative dielectric constant of silver is given by the Drude -Lorentz model(Dionne et al. 2006) as

$$
\varepsilon_{m}=\varepsilon_{\infty}-\frac{\omega_{p}^{2}}{\omega^{2}-i \omega \gamma^{\prime}}
$$

Here, $\varepsilon \infty, \gamma$, and $\omega \mathrm{p}$ are the dielectric constant at the infinite frequency, the electron collision frequency, the bulk plasma frequency, respectively.

The parameters are set to be $\varepsilon \infty=3.7$, $\omega \mathrm{p}=9.1 \mathrm{eV}, \gamma=0,018$ (Al Mahmod et al. 2018). During the FDTD simulations, only a single propagation mode TM can exist in the structure since the wavelength of the incident light is greater than the width of the waveguide. Therefore, the TM-polarized plane wave throws the MIM structure, the incident light will be coupled into the waveguide, and SPP waves are formed on the two metal interfaces, the dispersion relation of the fundamental TM model in the plasmonics waveguide structure is given by(Dionne et al. 2006). The fundamental TM mode of the plasmonic waveguide is excited by a plane wave whose amplitude is set as 1 incident from the input waveguide(Kazanskiy et al. 2019), the monitor is set at the output of the MIM waveguide as it in Figure. 1(a) to detect the transmittance as $\mathrm{T}=$ Pout/Pin, the incident power of Pin and transmitted power of Pout are monitored and calculated during the simulation.

As known, the accumulated phased shift per round trip in a plasmonics resonator for the SPPs is expressed as $\varphi=4 \pi$ neff S $/ \lambda+2 \phi$ (U. Fano 1961), Based on the standing wave theory, the constructive interference should occur when $\phi=2 \mathrm{~N} \pi$, thus, the resonance wavelength is determined by:

$$
\lambda=\frac{2 n_{e f f} H}{(N-\varphi / \pi)}
$$

Where $\mathrm{H}$ is the effective length of the resonator from the SPP incident position to that where the reflection occurs, and neff denotes the real part of the effective refractive index of the SPPs, $N$ indicates resonance orders in the square resonator or the cavity and $\varphi$ is the phase shift brought by the SPP reflection off the metal wall in the resonator.

\section{Numerical results and discussion}

A two-dimensional FDTD method used to analyze the structure by using RSoft CADsoftware(Zhang et al. 2009), with a perfectly matched layer (PML) absorbing boundary condition in $\Delta x=\Delta z=0.5 \mathrm{~nm} x$ and, and the time step is set as: $c \Delta t \leq 1 /(\operatorname{Square}((\Delta \mathrm{x})-2+(\Delta \mathrm{z})-2))(\mathrm{Wu}$ et al. 2014), where the $\mathrm{c}$ is the speed of light in the free space. The input type is Gaussian modulated continuous wave of TM polarization field, and by monitoring the output P2 we detect the transmission spectrum of the structure. The proposed structure's geometrical parameters L1, L3, L4, S, W' , are set as 455nm, 500nm, 450nm, $15 \mathrm{~nm}$ and $70 \mathrm{~nm}$, respectively, the width W of the MIM is set as 50nm. First, the MIM and the cavities' structure filled with nothing; this means that the insulator is air $(n=1)$. 


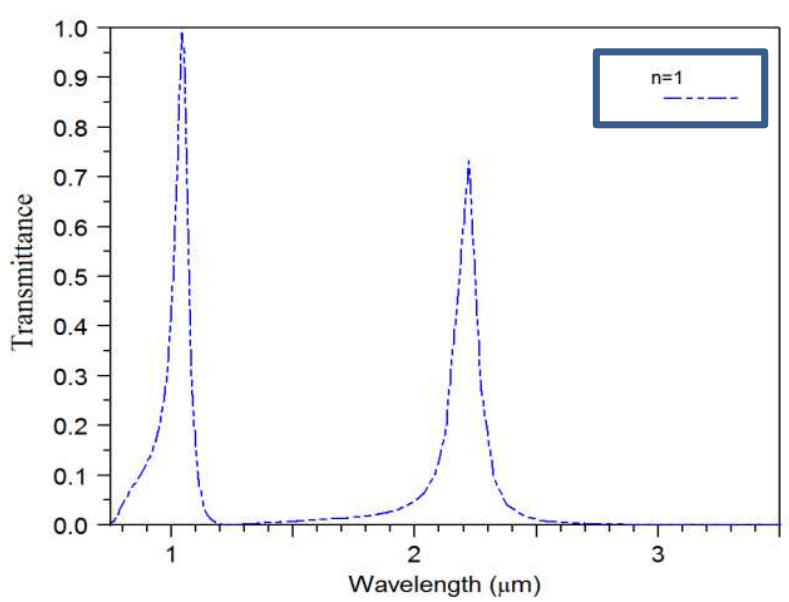

(a)

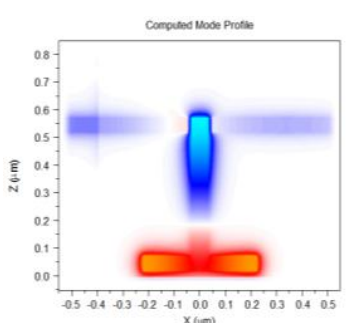

(b)

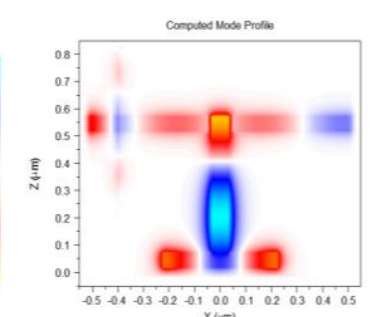

(c)

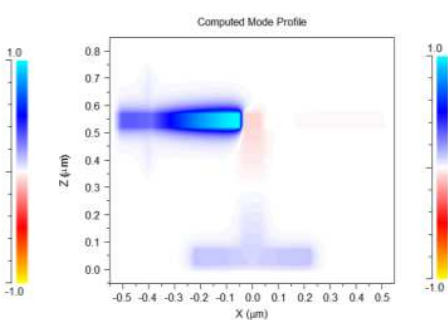

(d)

Fig. 2 The transmission spectra of the MIM waveguide(a). The contour profiles of field $\mid \mathrm{Hzl}$ of the resonator for(b) at $1.098 \mu \mathrm{m}$, for (c) at $1.297 \mu \mathrm{m}$, for (d) at 3 um.

In this case, two sharp Fano resonance appears according to Figure. 2(a), with a value of $98 \%$ and $67 \%$ of the transmission, named as mode 1 and mode 2 at the resonance wavelength of $1062 \mathrm{~nm}$ and $2272 \mathrm{~nm}$, respectively. In such circumstances, strong energy distributions can be observed in the output of the waveguide as it shown in Figure. 2(a,b); clearly we can notice an increase in the resonance wavelength is obtained at $1098 \mathrm{~nm}$ and $1297 \mathrm{~nm}$, corresponding the resonant peak wavelengths at the mode 1 and the mode 2 , respectively, the power transmission spectrum and IHyl field pattern at the resonance wavelength are achieved, on the other hand in Figure. 2(c) there is no SPP waves pass through the output waveguide at $3000 \mathrm{~nm}$, indicating that the wavelength cannot transport in this structure, which conforms with what we found in Figure. 2(a).

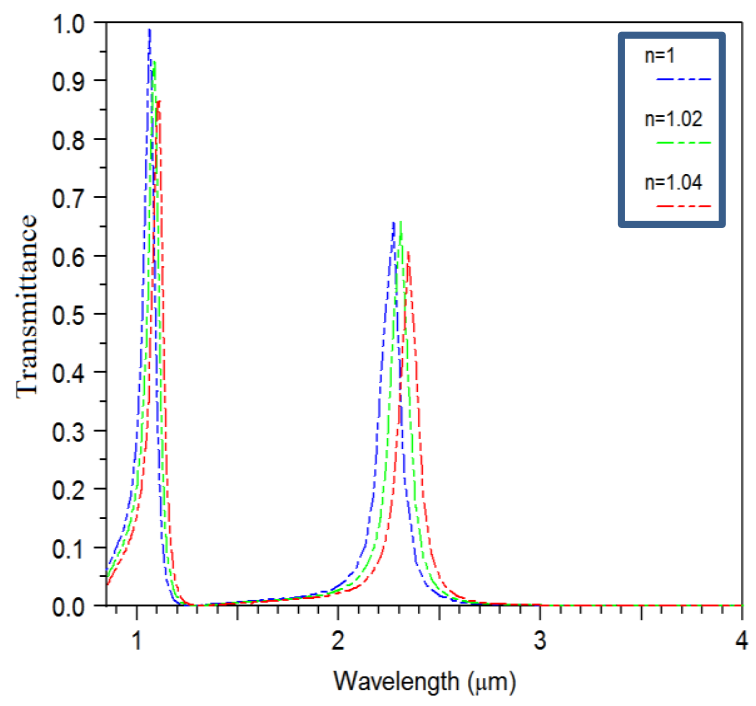

Fig. 3 The transmission spectra of the structure for different refractive indices $n$ ranged from 1.00 to 1.04 with step of 0.02 . 
Moreover, the transmission spectra of the structure with different refractive indices from 1.00 to 1.04 with step of 0.02 are studied, and the results are displayed in Figure. 3, it can be seen that the resonance wavelength at both modes shifting as we increase the refractive indices, this shift phenomenon occurs due to the proportional relation between the Re(neff) and $\lambda$ according to equation (3). When the refractive index of the structure is changed, this change in the resonant wavelength $(\Delta \lambda)$ provides information about the RI shift $(\Delta \mathrm{n})$. To this, it is conventional to define the spectral sensitivity of such sensors as $\mathrm{S}=\Delta \lambda / \Delta \mathrm{n}$ (Liu et al. 2010). Obviously, according to the definition, the sensitivity value of the plasmonics sensor achieved of model as $1155 \mathrm{~nm} / \mathrm{RIU}$, while mode 2 is $2360 \mathrm{~nm} / \mathrm{RIU}$. All sensors must be assessed concerning their sensitivity(Kwon 2013).

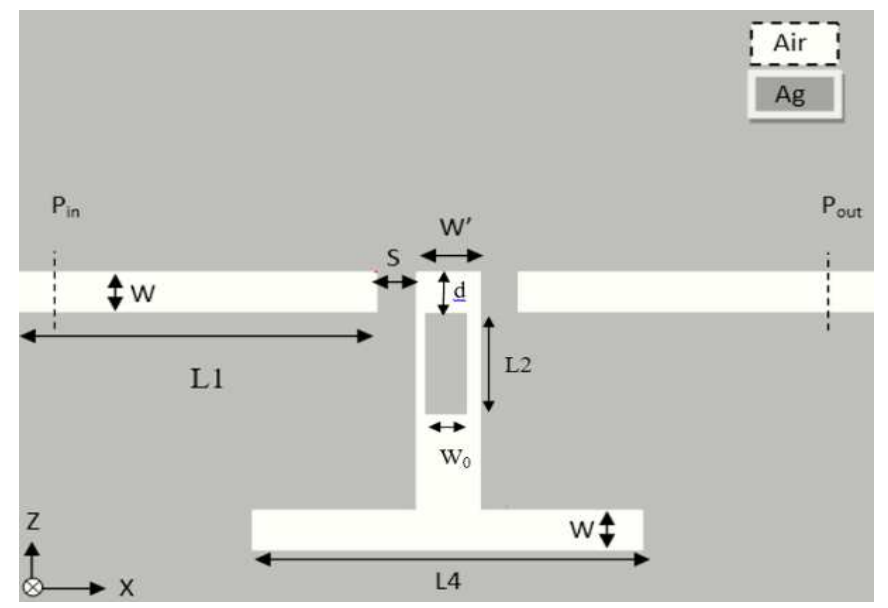

Fig. 4 Schematic diagram of the plasmonics sensor with cavity $\mathrm{C}$ (a).

Furthermore, to enhance the sensitivity we use a new cavity $\mathrm{C}$ inside the cavity A with unique parameters as shown in Figure. 4; we fix $\mathrm{S}$ and set L2, L3, L4, as $15 \mathrm{~nm}, 100 \mathrm{~nm}$, and $500 \mathrm{~nm}, 450 \mathrm{~nm}$, respectively. The transmission spectrum of both structure with and without cavity $\mathrm{C}$ is shown in Figure. 5(a), it can be seen that mode 1 and mode 2 have a linear green shift, the variations of resonant wavelengths of mode 1 and mode 2 are $293 \mathrm{~nm}$ and $229 \mathrm{~nm}$, respectively.

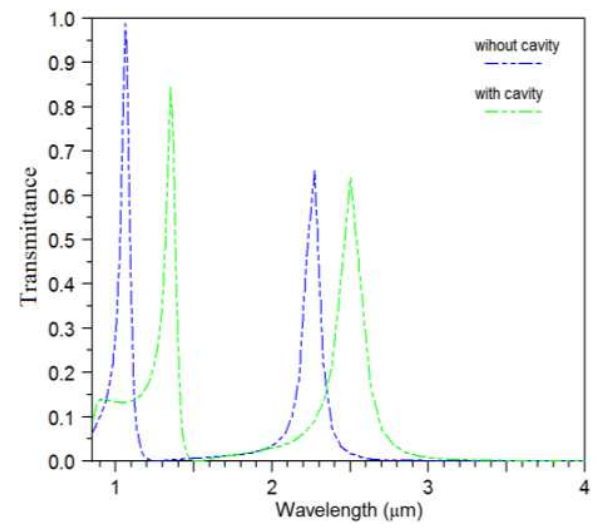

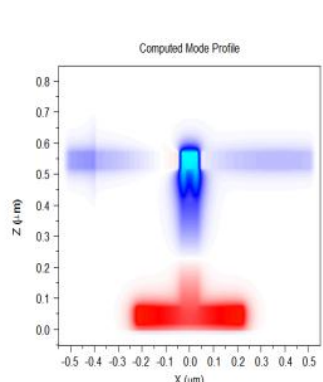

(b)

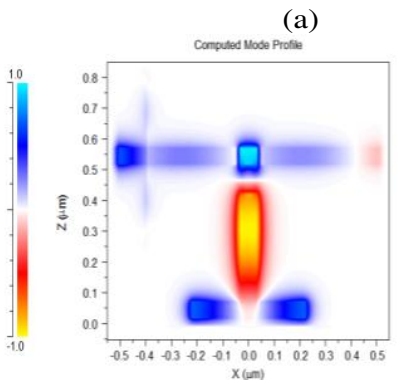

(c)

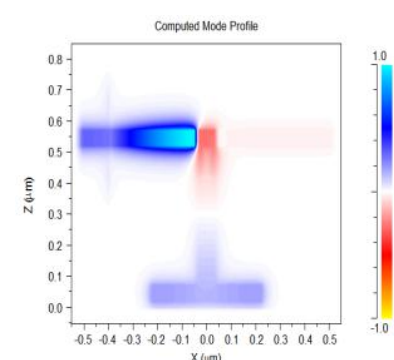

(d)

Fig. 5 The structure with and without cavity $C$ using L4=450, d=0.5 (b). The contour profiles of field IHzl of the resonator for (c1) at $1.393 \mu \mathrm{m}$. for (c2) at $2.587 \mu \mathrm{m}$. for (c3) at $3 \mu \mathrm{m}$. 
The results imply that with cavity $\mathrm{C}$ we decrease transmission from mode 1 and mode 2 by $14.14 \%$ and $2.6 \%$, respectively, however we improve and increasing the sensitivity with cavity $\mathrm{C}$. To gain a better understanding of the inner mechanism of SPRs in the MIM and the change in the transmission spectra, the magnetic field distribution of the spectra applied at the resonance presented in Figure. 5(b,c), a strong energy distribution can be observed shown in $\mathrm{c} 1$ and $\mathrm{c} 2$ correspond the resonance of mode 1 at $1.393 \mu \mathrm{m}$ and $2.587 \mu \mathrm{m}$ for mode 2 , however, for $\mathrm{b} 3$ at $3 \mu \mathrm{m}$ we notice that there is no resonance in output and this conform with what we found in Figure. 5(d). In order to further investigate the performance of the cavity $\mathrm{C}$ on the proposed structure of the plasmonic sensor, we studied the effect of the length $\mathrm{d}$ on the transmittance characteristics, by changing length $\mathrm{d}$ from 0 to $200 \mathrm{~nm}$ with step of $50 \mathrm{~nm}$, we notice that the position of cavity $\mathrm{C}$ has an effect on the narrow and the transmission on both modes as shown in Figure. 6(a). Clearly it is observed that the transmission spectra of the peak for $\mathrm{d}=100 \mathrm{~nm}$ become better and narrow and when we increase the length $\mathrm{d}=150,200 \mathrm{~nm}$, we decrease the transmission of both modes, however, by using the value of $\mathrm{d}=0$ the Fano resonance loss their narrow and transmission. Therefore, we used d of $50 \mathrm{~nm}$ for the rest of our studies to have a narrowest resonant peak for better resolution. Next, we focus on the influence of the refractive indices by increasing $\mathrm{n}$ from 1.00 to 1.04 with step of 0.02 , as it displayed in Figure. 6(b), we notice a redshift in the resonance wavelength by increasing the refractive index, this change in the resonant wavelength $\Delta \lambda$ provides a highly sensitive for both modes, therefore we achieved the sensitivity for mode 1 and mode 2 as $1166 \mathrm{~nm} / \mathrm{RIU}, 2220$ $\mathrm{nm} / \mathrm{RIU}$, respectively.

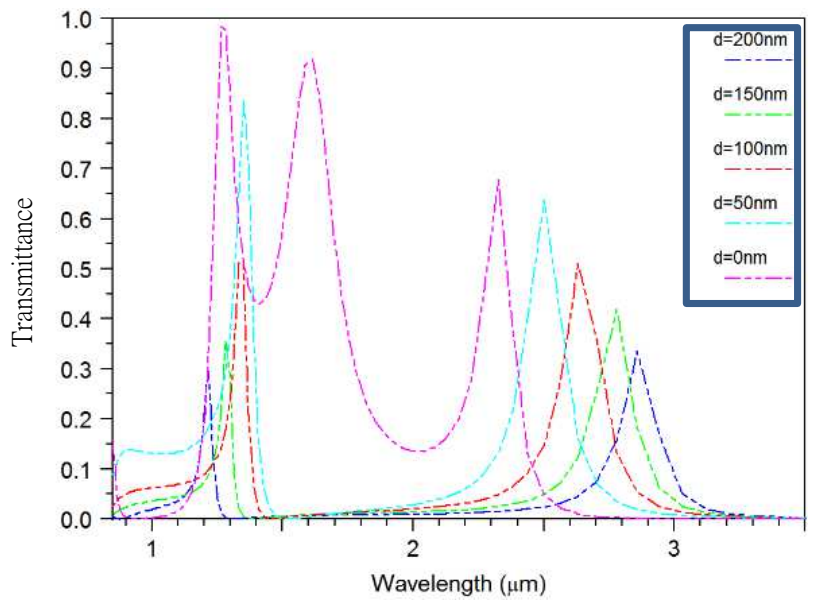

(a)

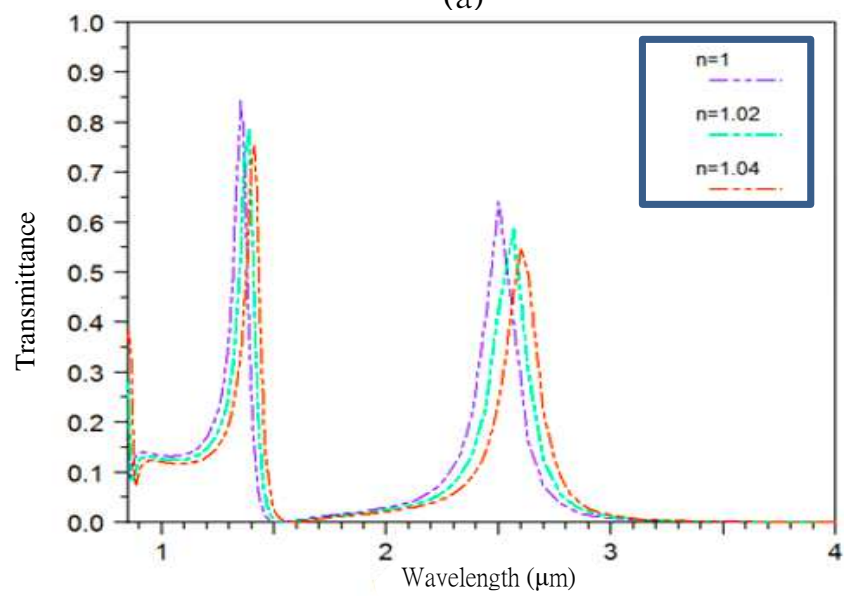

(b)

Fig. 6 The Shift of the resonance wavelength with cavity $C$ by changing length d from 0 to $200 \mathrm{~nm}$ (a). The transmission spectra of the structure for different refractive indices $n(b)$.

To improve the performance of the sensor, we also investigate how the length L4 affects on the sensing characteristics and the dependence of resonance wavelength, we varied the value of L4 from 100 to $700 \mathrm{~nm}$ with a step of $100 \mathrm{~nm}$ and 70nm as W' . 


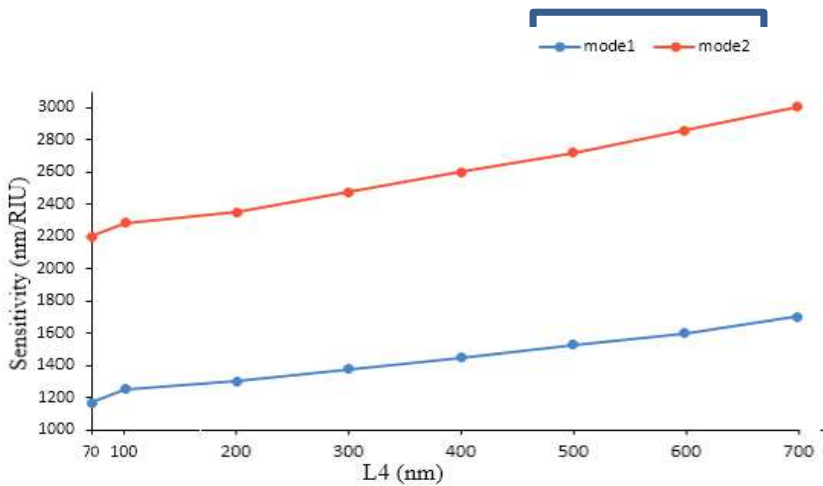

(a)

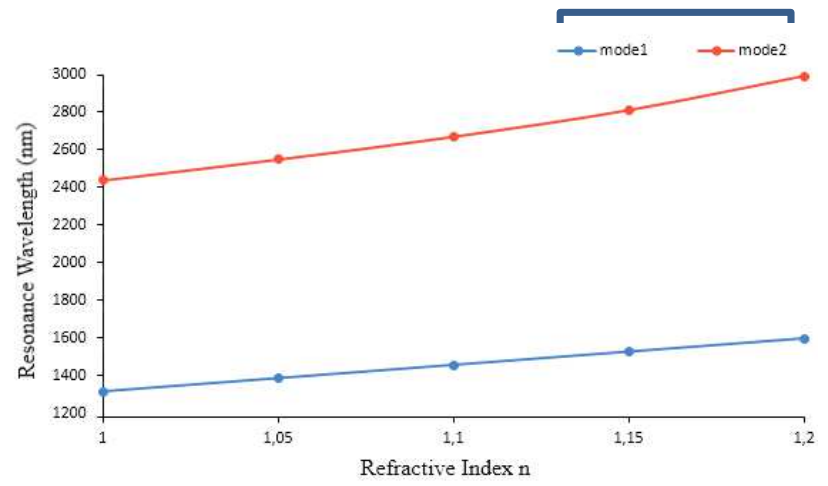

(b)

Fig. 7 The sensitivity versus the rectangular cavity $\mathrm{C}$ length L4(a). The resonance wavelengths versus different refractive indices $n$ by varing $n$ from 1 to 1.2 with a step of $0.05(\mathrm{c})$.

Indeed, we noticed a significant linear relation between the sensitivity and the value of the length L4, by increasing L4 we also increase S for both modes as shown Figure 7(a). Further, the sensitivity for mode 1 increased from 1166 to $1650 \mathrm{~nm} / \mathrm{RIU}$, then we archive highly sensitivity from 2220 to $3010 \mathrm{~nm} / \mathrm{RIU}$ for mode 2 , this sensitivity value of the structure is higher than that has been obtained in Table.1.

\begin{tabular}{c|c|c}
\hline \hline Référence & $\begin{array}{c}\text { Sensitivity } \\
(\mathrm{nm} / \mathrm{RIU})\end{array}$ & year \\
\hline $\begin{array}{c}\text { (Ben salah et } \\
\text { al. 2019) }\end{array}$ & 2602,5 & 2018 \\
\hline $\begin{array}{c}\text { (Butt et al. } \\
\text { 2019) }\end{array}$ & 1367 & 2019 \\
\hline $\begin{array}{c}\text { (Chao et al. } \\
\text { 2020) }\end{array}$ & 2080 & 2020 \\
\hline $\begin{array}{c}\text { (Achi et al. } \\
\text { 2020) }\end{array}$ & 23807 & 2020 \\
\hline $\begin{array}{c}\text { (Butt et al. } \\
\text { 2020) }\end{array}$ & 1948,67 & 2020 \\
\hline $\begin{array}{c}\text { (Nejat and } \\
\text { Nozhat 2020) }\end{array}$ & 2000 & 2020 \\
\hline In this work & 3010 & 2020 \\
\hline
\end{tabular}

Table. 1: Sensitivity comparison of different sensor structures.

Furthermore, according to Figure. 7(b) by varying $\mathrm{n}$ from 1 to 1.2 with a step of 0.5 a linear relationship between the RI and the resonance wavelength achieved, this due to the correlation relation between the effective RI and the resonance wavelength in Equation (3), Moreover, the resonant wavelength is inversely proportional with the effective resonance length.

Therefore, the calculated results show that the sensitivity can be improved 
by increasing L4. The high sensitivity achieved in our proposed sensor with improving the transmission level affords extra features for designing real-time on-chip optical sensors.

\section{Conclusion}

We proposed and analyzed a simple design for a sensor structure based on MIM rectangular cavity by using the FDTD method, the simulation results show two modes and the resonance wavelength shifted linearly by increasing the length of the cavity T. Further, by engineering the length of the cavity $\mathrm{C}$ we achieved a sensitivity of RI sensor is reaching to $1600 \mathrm{~nm}$.RIU-1 and 3010 nm.RIU-1 for mode 1 and mode 2, respectively.

In addition to the plasmonic sensor application, the structure can also be applied to optical filters and with a high sensitivity, simple and compact structure this sensor has extensive potential in biosensing and optical on-chip nano-sensors. Relatively easy fabrication, simple configuration, and compact structure. 


\section{References}

Achi, S.E., Hocini, A., Salah, H.B., Harhouz, A.: REFRACTIVE INDEX SENSOR MIM BASED WAVEGUIDE COUPLED WITH A SLOTTED SIDE RESONATOR. 96, $147-156(2020)$

Butt, M.A., Kazanskiy, N.L., Khonina, S.N.: Highly integrated plasmonic sensor design for the simultaneous detection of multiple analytes. Curr. Appl. Phys. 20, 1274 - 1280 (2020). https://doi.org/10.1016/j.cap.2020.08.020

Butt, M.A., Khonina, S.N., Kazanskiy, N.L.: Plasmonic refractive index sensor based on metal - insulator-metal waveguides with high sensitivity. J. Mod. Opt. 66, 1038 1043 (2019). https://doi.org/10.1080/09500340.2019.1601272

Chao, C.T.C., Chau, Y.F.C., Huang, H.J., Kumara, N.T.R.N., Kooh, M.R.R., Lim, C.M., Chiang, H.P.: Highly sensitive and tunable plasmonic sensor based on a nanoring resonator with silver nanorods. Nanomaterials. 10, 1 - 14 (2020). https://doi.org/10.3390/nano10071399

Dionne, J.A., Sweatlock, L.A., Atwater, H.A., Polman, A.: Plasmon slot waveguides: Towards chip-scale propagation with subwavelength-scale localization. Phys. Rev. B Condens. Matter Mater. Phys. 73, (2006). https://doi.org/10.1103/PhysRevB.73.035407

Fano, U.: Effects of Configuration Interaction on Intensities and Phase Shifts. Phys. Rev. 124, 12 (1961)

Han, Z. Liu, L. Forsberg, E.: Ultra-compact directional couplers and Mach-Zehnder interferometers employing surface plasmon polaritons. Opt. Commun. 25, 690 - 69 (2006)

Hocini, A., Ben salah, H., Khedrouche, D., Melouki, N.: A high-sensitive sensor and band-stop filter based on intersected double ring resonators in metal - insulatormetal structure. Opt. Quantum Electron. 52, 1-10 (2020). https://doi.org/10.1007/s11082-020-02446-x

Huang, Y.-W., Chen, W.T., Wu, P.C., Fedotov, V., Savinov, V., Ho, Y.Z., Chau, Y.-F., Zheludev, N.I., Tsai, D.P.: Design of plasmonic toroidal metamaterials at optical frequencies. Opt. Express. 20, 1760 (2012). https://doi.org/10.1364/oe.20.001760

Kazanskiy, N.L., Khonina, S.N., Butt, M.A.: Plasmonic Sensors Based onMetal-InsulatorMetal Waveguides for Refractive Index Sensing Applications: A Brief Review. Phys. E Low-dimensional Syst. Nanostructures. (2019). https://doi.org/10.1016/j.physe.2019.113798

Kwon, S.H.: Deep subwavelength-scale metal-insulator-metal plasmonic disk cavities for refractive index sensors. IEEE Photonics J. 5, 4800107 (2013). https://doi.org/10.1109/JPHOT.2013.2244206

Liu, N., Mesch, M., Weiss, T., Hentschel, M., Giessen, H.: Infrared perfect absorber and its application as plasmonic sensor. Nano Lett. 10, $2342-2348$ (2010). https://doi.org/10.1021/n19041033

Al Mahmod, M.J., Hyder, R., Islam, M.Z.: A Highly Sensitive Metal-Insulator-Metal Ring Resonator-Based Nanophotonic Structure for Biosensing Applications. IEEE Sens. J. 18, 6563 - 6568 (2018). https://doi.org/10.1109/JSEN.2018.2849825

Miroshnichenko, A.E., Flach, S., Kivshar, Y.S.: Fano resonances in nanoscale structures. Rev. Mod. Phys. 82, 2257-2298 (2010). https://doi.org/10.1103/RevModPhys.82.2257

Nejat, M., Nozhat, N.: Multi-band MIM refractive index biosensor based on Ag-air grating with equivalent circuit and T-matrix methods in near-infrared region. Sci. Rep. 10, 1 - 12 (2020). https://doi.org/10.1038/s41598-020-63459-w

Ren, W., Dai, Y., Cai, H., Ding, H., Pan, N., Wang, X.: Tailoring the coupling between localized and propagating surface plasmons: realizing Fano-like interference and highperformance sensor. Opt. Express. 21, 10251 (2013). https://doi.org/10.1364/oe.21.010251

Reza, M., Mansouri-birjandi, M.A.: A high-sensitivity sensor based on three-dimensional metal - insulator - metal racetrack resonator and application for hemoglobin detection. Photonics Nanostructures - Fundam. Appl. 32, 28 - 34 (2018). https://doi.org/10.1016/j.photonics.2018.08.002

Ben salah, H., Hocini, A., Temmar, M.N., Khedrouche, D.: Design of mid infrared high sensitive metal-insulator-metal plasmonic sensor. Chinese J. Phys. 61, 86 - 97 (2019). https://doi.org/10.1016/j.cjph.2019.07.006

U. Fano: Effects of Configuration Interaction on Intensities and Phase Shifts. Phys.Rev. 124, 13 (1961)

Wu, T., Liu, Y., Yu, Z., Peng, Y., Shu, C., Ye, H.: The sensing characteristics of 
plasmonic waveguide with a ring resonator. Opt. Express. 22, 7669 (2014). https://doi.org/10.1364/oe.22.007669

Xiang, D. Li, W.: MIM Plasmonic Waveguide Splitter with Tooth-Shaped Structures. J. Mod. Opt. 61, 222 - 226 (2014)

Yi, X., Tian, J., Yang, R.: Tunable Fano resonance in MDM stub waveguide coupled with a U-shaped cavity. Eur. Phys. J. D. 72, (2018). https://doi.org/10.1140/epjd/e201880734-6

Zhang, Q., Huang, X.-G., Lin, X.-S., Tao, J., Jin, X.-P.: A subwavelength coupler-type MIM optical filter. Opt. Express. 17, 7549 (2009). https://doi.org/10.1364/oe.17.007549

Zhao, W. Lu, Z.: Nanoplasmonic Optical Switch Based on Ga-Si 3 N 4-Ga Waveguide.., 2011, 50, . Opti.l Engin. 50, 074002 (2011) 
Figures

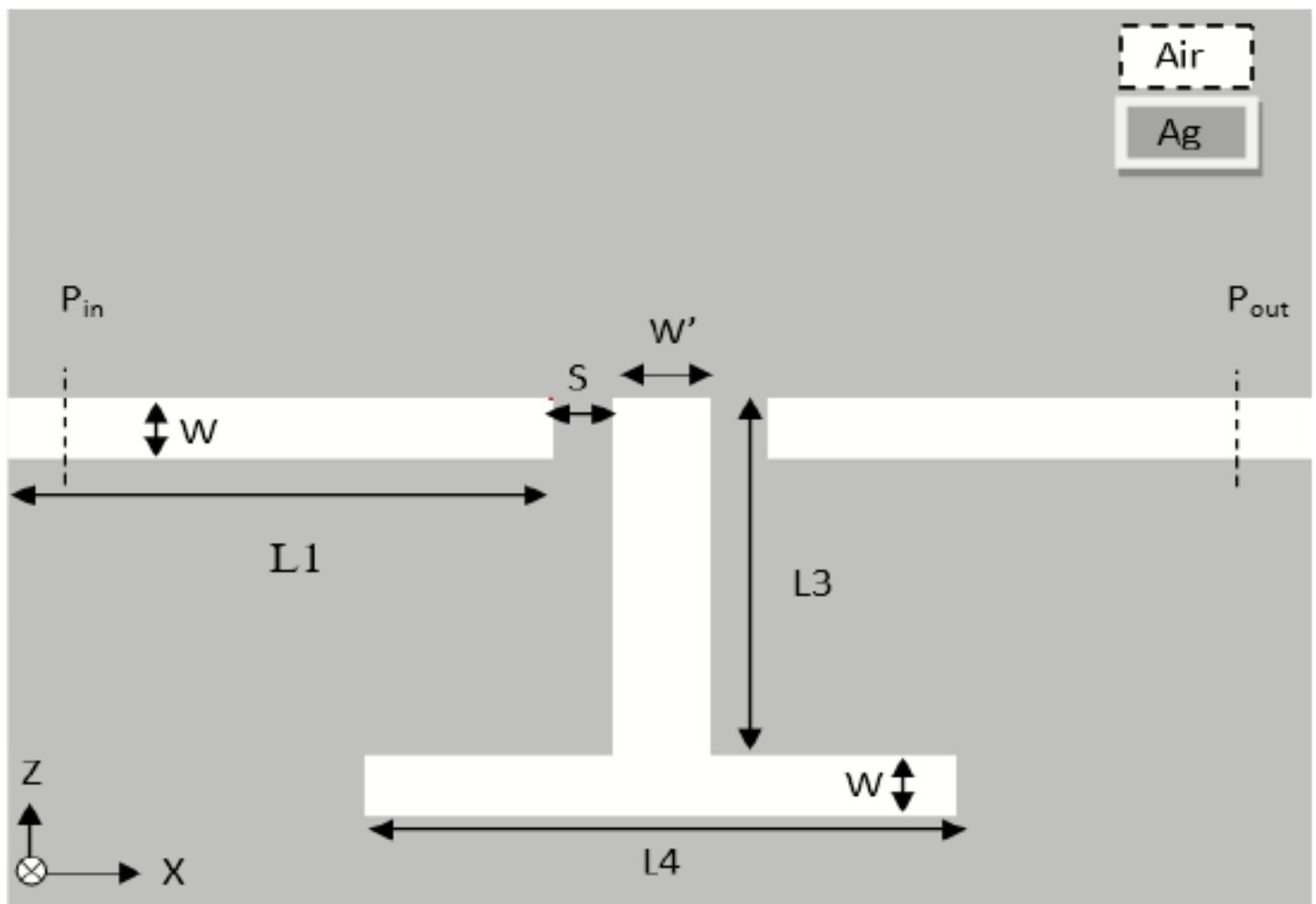

Figure 1

2D schematic diagram of the proposed sensor structure. 


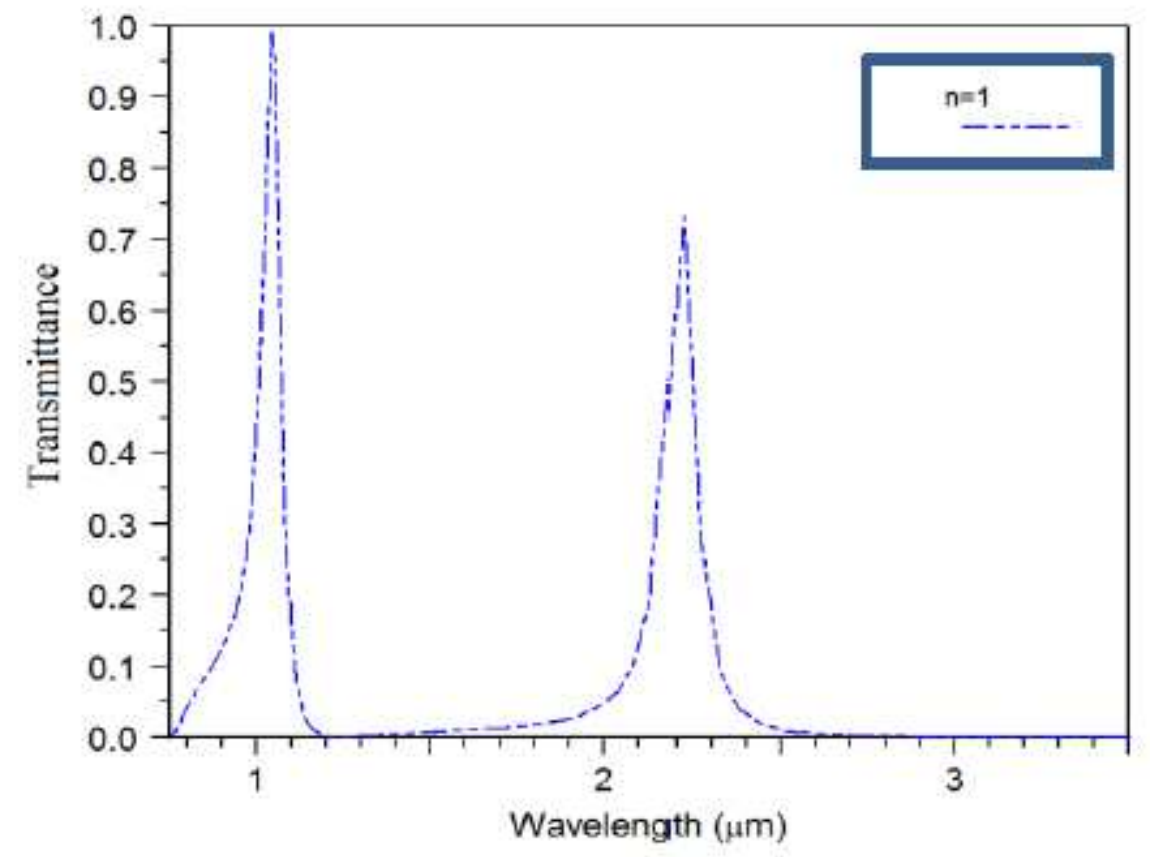

(a)

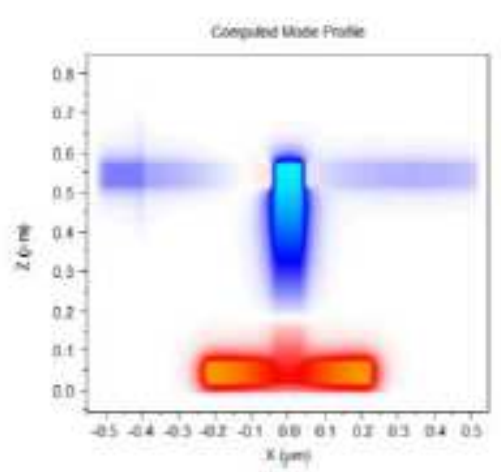

(b)

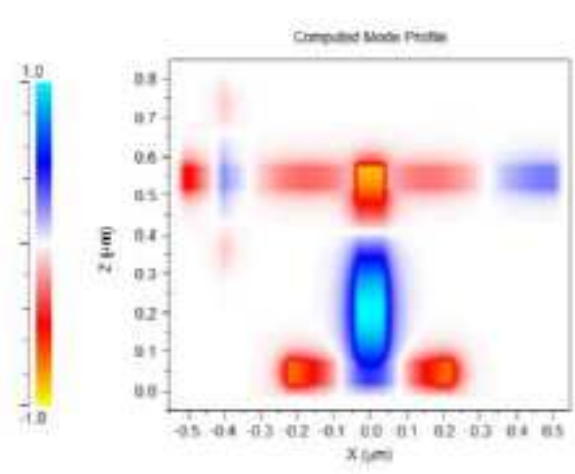

(c)
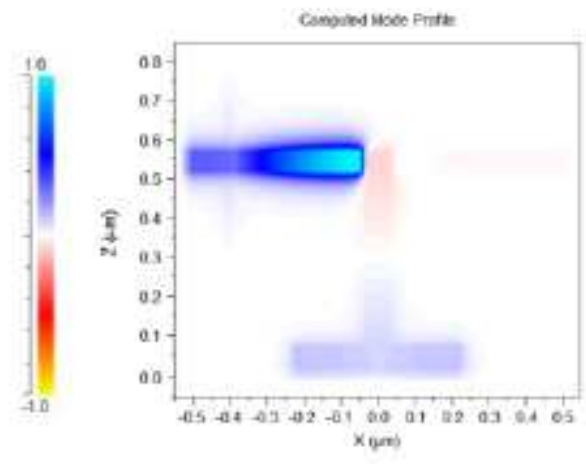

(d)

\section{Figure 2}

The transmission spectra of the MIM waveguide(a). The contour profiles of field $|\mathrm{Hz}|$ of the resonator for(b) at $1.098 \mu \mathrm{m}$, for (c) at $1.297 \mu \mathrm{m}$, for (d) at $3 \mu \mathrm{m}$. 


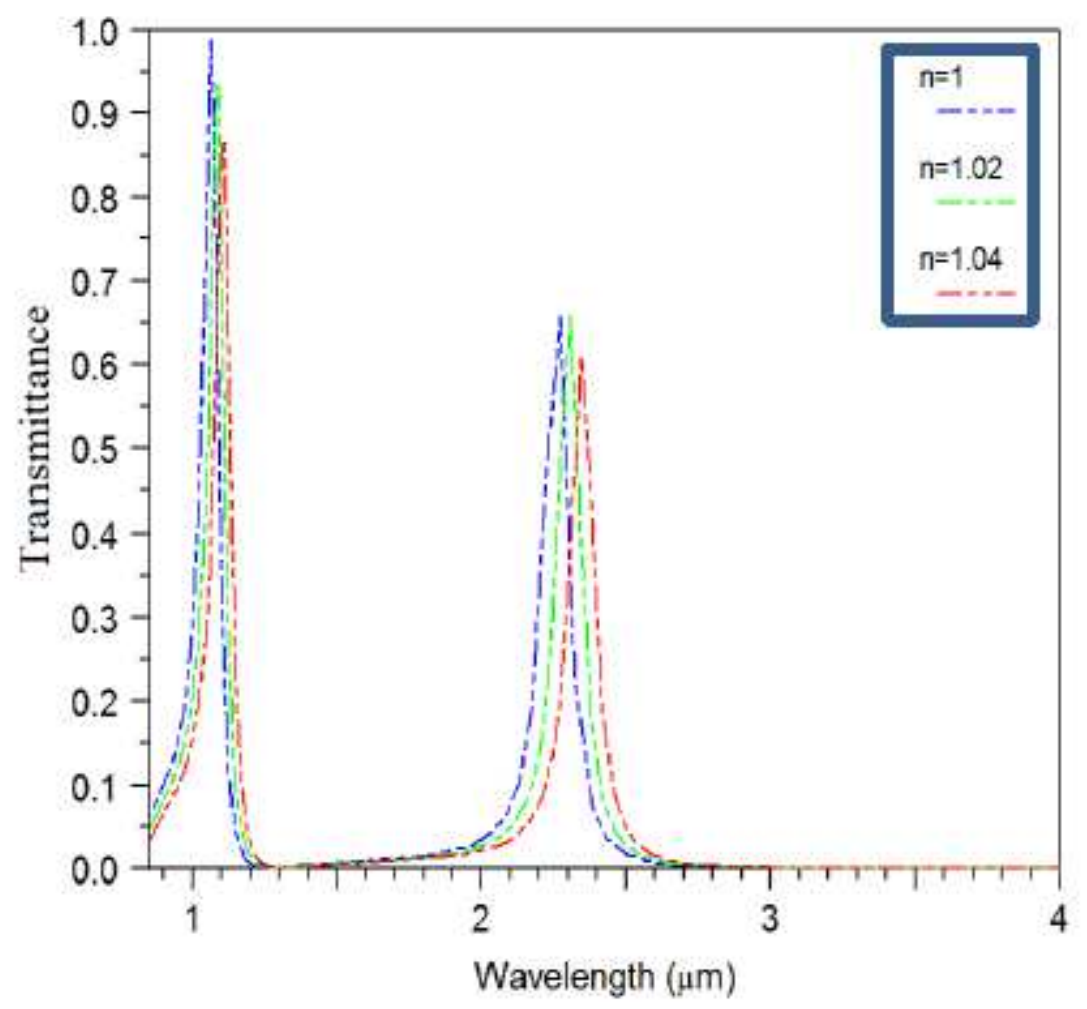

Figure 3

The transmission spectra of the structure for different refractive indices $\mathrm{n}$ ranged from 1.00 to 1.04 with step of 0.02 .

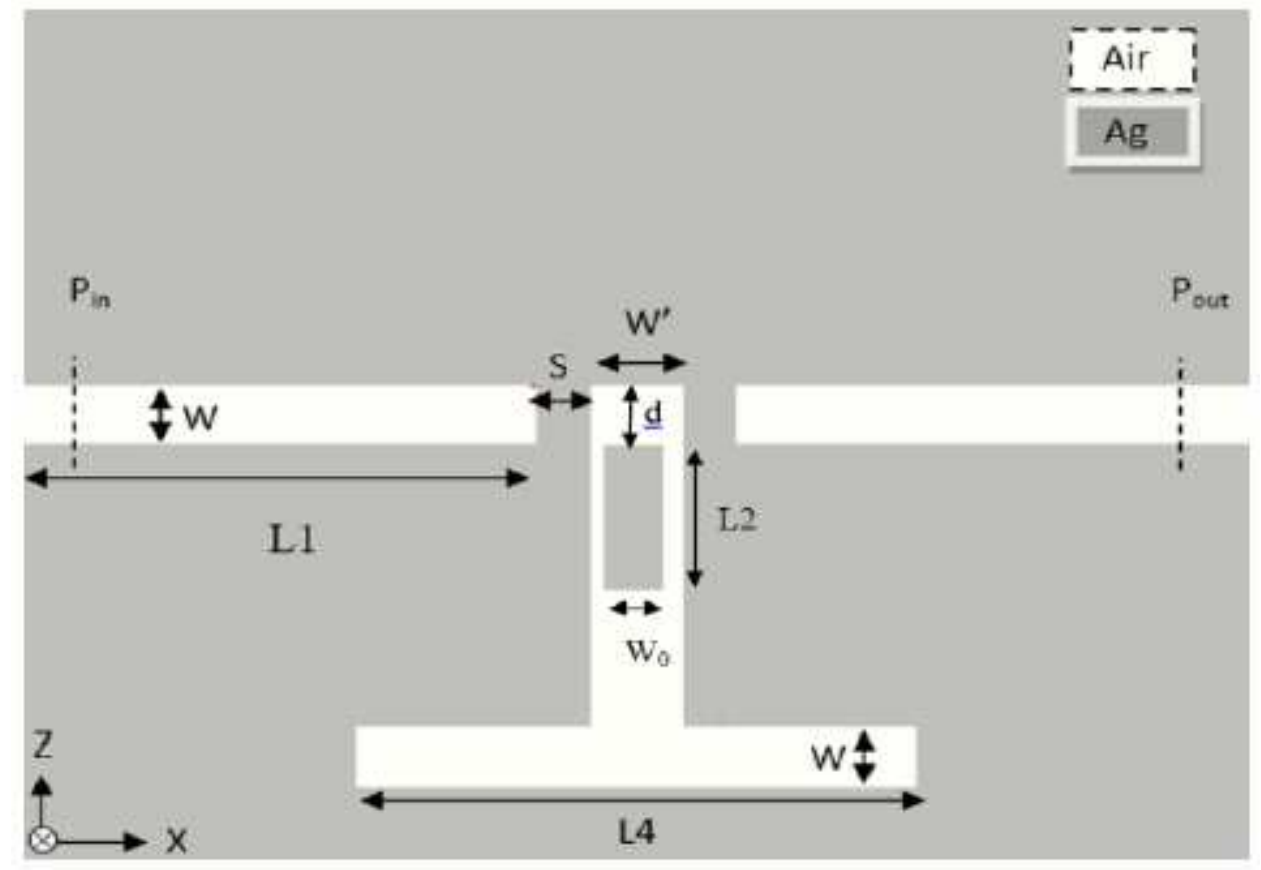

Figure 4

Schematic diagram of the plasmonics sensor with cavity C (a). 


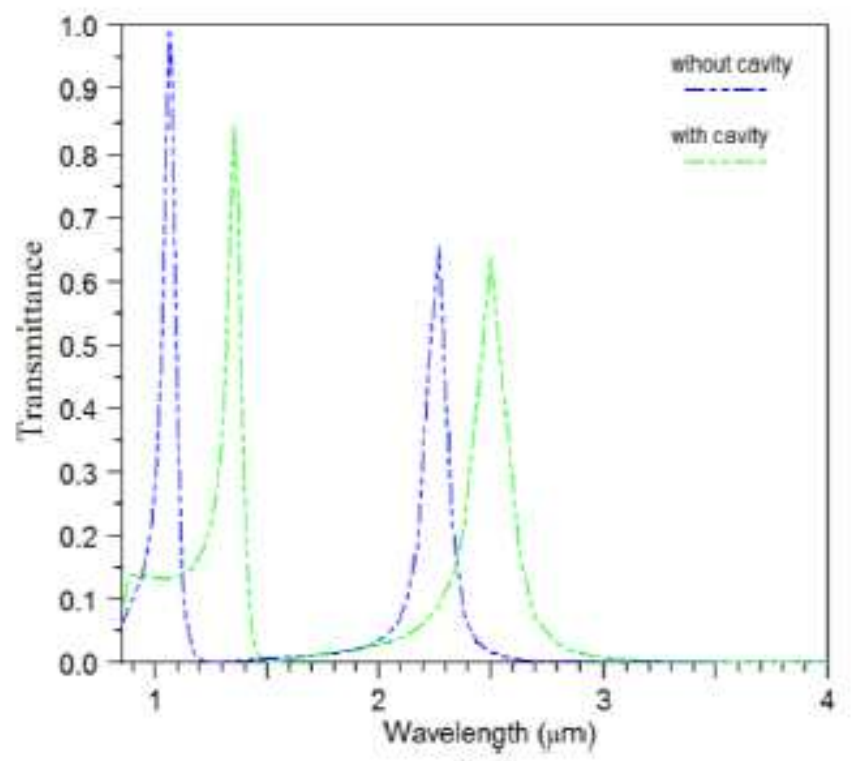

(a)

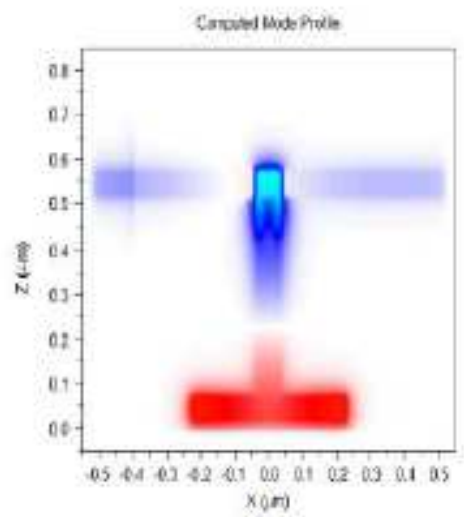

(b)

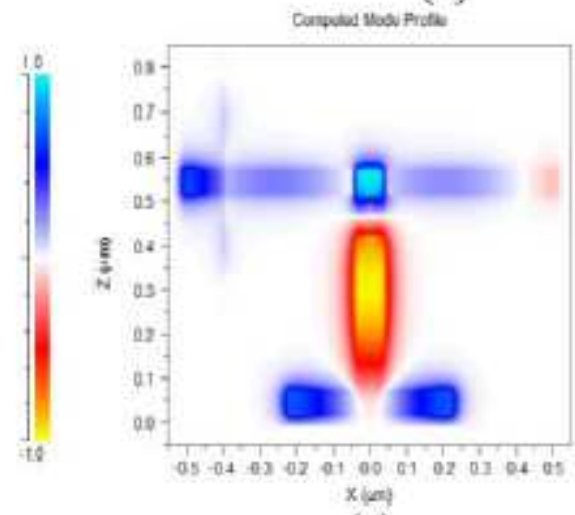

(c)

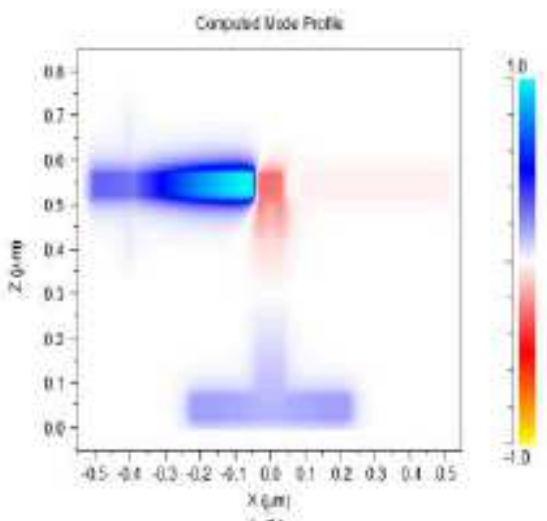

(d)

\section{Figure 5}

The structure with and without cavity $C$ using $L 4=450, d=0.5$ (b). The contour profiles of field $|\mathrm{Hz}|$ of the resonator for (c1) at $1.393 \mu \mathrm{m}$. for (c2) at $2.587 \mu \mathrm{m}$. for (c3) at $3 \mu \mathrm{m}$. 

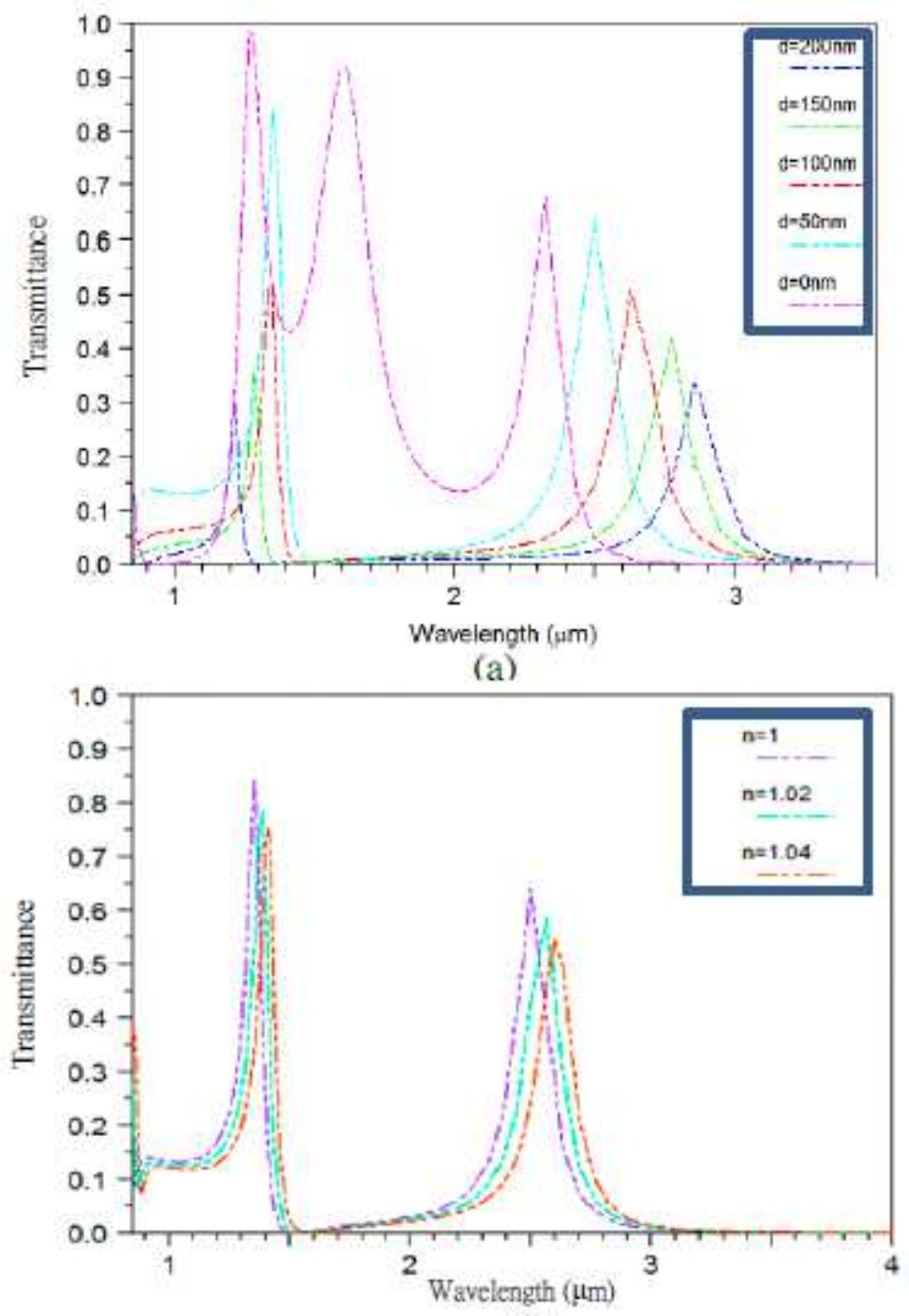

(b)

Figure 6

The Shift of the resonance wavelength with cavity $C$ by changing length $d$ from 0 to $200 \mathrm{~nm}$ (a). The transmission spectra of the structure for different refractive indices $n(b)$. 


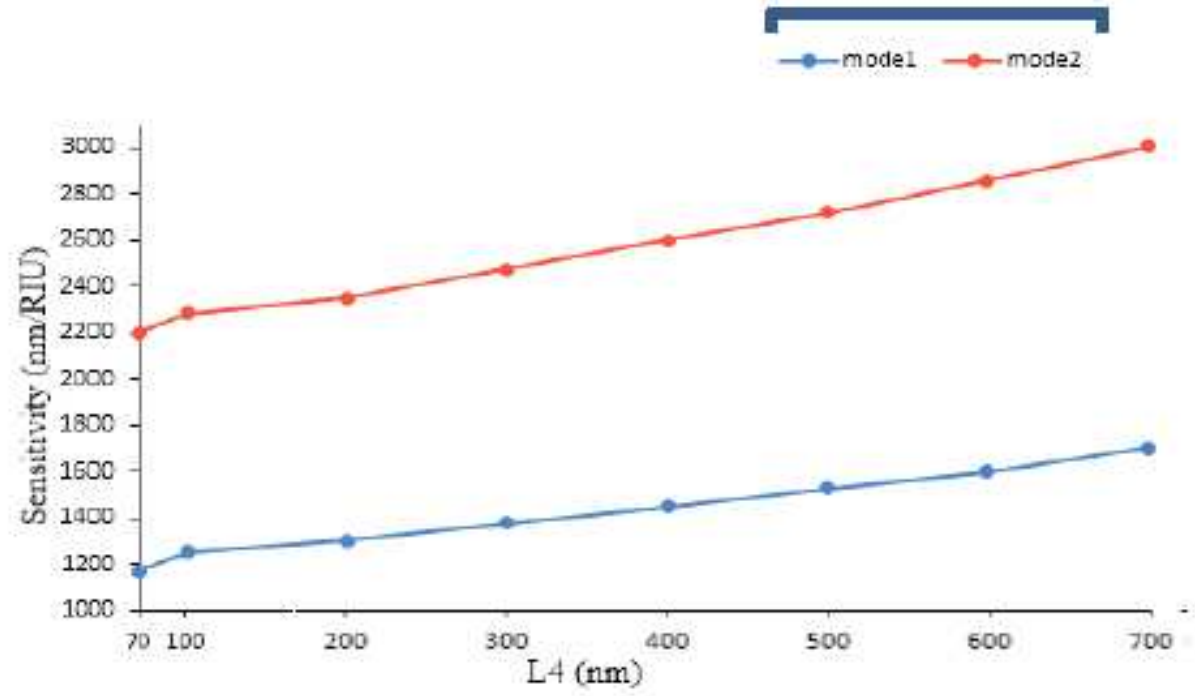

(a)

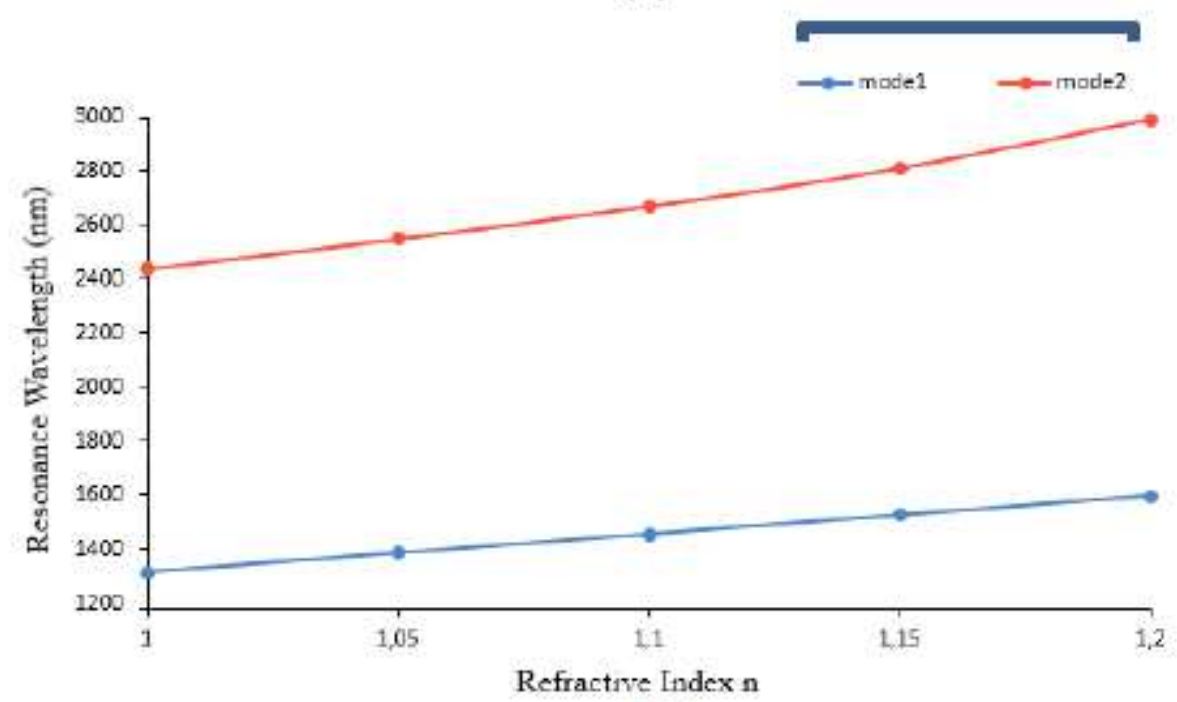

(b)

\section{Figure 7}

The sensitivity versus the rectangular cavity $C$ length $L 4(a)$. The resonance wavelengths versus different refractive indices $n$ by varing $n$ from 1 to 1.2 with a step of 0.05 (c). 